\title{
Detailed Solution of a System of Singular Integral Equations for Mixed Mode Fracture in Functionally Graded Materials
}

\author{
Youn-Sha Chan ${ }^{1}$, Edward Athaide ${ }^{2}$, Kathryn Belcher ${ }^{3}$ \& Ryan Kelly ${ }^{4}$ \\ ${ }^{1}$ Department of Mathematics and Statistics, University Houston-Downtown, Houston, USA \\ 2 Department of Applied Mathematics, Harvard College, Cambridge, MA 02138, USA \\ ${ }^{3}$ Department of Mathematics, Fordham University, Bronx, NY 10458, USA \\ 4 Department of Mechanical, Aerospace, and Biomedical Engineering, The University of Tennessee, Knoxville, TN \\ 37996, USA \\ Correspondence: Youn-Sha Chan, Department of Mathematics and Statistics, University Houston-Downtown, One Main \\ Street, Houston, Texas 77002, USA. E-mail: chany@uhd.edu
}

Received: November 3, 2019 Accepted: December 31, 2019 Online Published: January 15, 2020

doi:10.5539/jmr.v12n1p43 URL: https://doi.org/10.5539/jmr.v12n1p43

\begin{abstract}
A mixed mode crack problem in functionally graded materials is formulated to a system of Cauchy singular Fredholm integral equations, then the system is solved by the singular integral equation method (SIEM). This specific crack problem has already been solved by N. Konda and F. Erdogan (Konda \& Erdogan 1994). However, many mathematical details have been left out. In this paper we provide a detailed derivation, both analytical and numerical, on the formulation as well as the solution to the system of singular Fredholm integral equations. The research results include crack displacement profiles and stress intensity factors for both mode I and mode II, and the outcomes are consistent with the paper by Konda \& Erdogan (Konda \& Erdogan 1994).
\end{abstract}

Keywords: systems of singular linear integral equations, functionally graded materials, crack-tip singularity, stress intensity factor

\section{Introduction}

\subsection{Motivation and Background}

In examining material failure, most failure processes (corrosion, wear, fatigue, etc.) appear to be at the material interface due to the sudden change of material composition. To cope with these failure issues, functionally graded materials (FGMs) are developed (Hirai, 1996; Kawasaki \& Watanabe, 1990; Kaysser, 1998; Koizumi, 1993; Bhavar, 2017; Heer \& Bandyopadhyay, 2018; Kuang et al., 2019). In FGMs, material compositions are engineered by controlling the material properties near and at the interface of the materials, so that material failures can be minimized. In terms of linear elasticity theory, isotropic homogeneous materials are governed by Navier equations (Evans, 1998; Sadd, 2005)

$$
\mu \Delta \overrightarrow{\mathbf{u}}(\mathbf{x})+(\lambda+\mu) \nabla(\nabla \cdot \overrightarrow{\mathbf{u}}(\mathbf{x}))=0
$$

where $\mu$ is the shear modulus, $\lambda$ is the Lamé constant, and $\overrightarrow{\mathbf{u}}$ is the displacement vector at position $\mathbf{x}=(x, y, z)$. Equation (1) is a system of second order linear partial differential equations (PDEs), and finding its analytical exact solution is a classical outcome (Debnath, 1995; Evans, 1998; Folland, 1992; Muskhelishvili, 1953; Muskhelishvili, 1963). However, the material nonhomogeniety of FGM changes the PDEs (1) to

$$
\mu(\mathbf{x}) \Delta \overrightarrow{\mathbf{u}}(\mathbf{x})+[\lambda(\mathbf{x})+\mu(\mathbf{x})] \nabla(\nabla \cdot \overrightarrow{\mathbf{u}}(\mathbf{x}))+\left(\nabla \overrightarrow{\mathbf{u}}(\mathbf{x})+\nabla \overrightarrow{\mathbf{u}}(\mathbf{x})^{T}\right) \nabla \mu(\mathbf{x})+(\nabla \cdot \overrightarrow{\mathbf{u}}(\mathbf{x})) \nabla \lambda(\mathbf{x})=0
$$

where $\mu$ and $\lambda$ are not constants any more but instead are functions of the material position $\mathbf{x}=(x, y, z)$. It is not a trivial task to obtain the exact analytical solution to PDEs (2); in general, numerical approximation is needed eventually in order to get the solution to PDEs (2).

Another issue arises if we are to solve a crack problem in FGMs: crack-tip singularity (Erdogan, 1995; Gu \& Asaro, 1997; Jin \& Batra, 1996). Due to the crack-tip singularity, commonly used numerical methods, such as finite element method or finite difference method, are not suitable for solving the crack problem. For instance, numerical convergence, stability, and accuracy at crack-tips become an issue when general numerical methods are used. The one particular method for 
solving a crack problem in linear elastic medium is called "singular integral equation method" (Erdogan \& Gupta, 1972; Erdogan, Gupta, \& Cook, 1973; Jin \& Noda, 1994; Kabir et al, 1998), or SIEM, and it is our main focus in this paper to give a detailed analysis, both analytical and numerical, on SIEM.

Some earlier work by Chan and Koshkin (Chan \& Koshkin, 2019) has already addressed the method SIEM in detail, and the analysis outcomes have been compared with the paper by Delale and Erdogan (Delale \& Erdogan, 1983) This earlier work is for a mode I crack, and the governing PDE is a scalar problem. Here we are solving a mixed mode (mode I and mode II) crack problem, and the governing PDE is a system as described in equation (2) above. To demonstrate the SIEM in detail for solving a mixed mode crack problem, we choose a very well-known paper by Konda and Erdogan (Konda \& Erdogan, 1994) with which to compare our results.

Although SIEM is often used to solve crack problems, the derivation is usually sparsely documented. Generally, when SIEM is used, the theoretical derivations are very brief or absent. Furthermore, to our best knowledge on literature survey, there has been little numerical programming publication on SIEM. This is another motivation for us to give a fully detailed analysis on SIEM. In addition to providing a detailed mathematical derivation of the analytical part, we also give a comprehensive explanation at each step of the numerical process. One of our primary contributions is the explanation and computation of the numerical solution steps. The theoretical details are provided in the paper, and the MATLAB files we used can be found at github.com/SolidMechanicsREU2019/MixedModeCrack. The files are free to access, download, and revise so that they can be used for solving other crack problems.

This research was conducted during the course of an REU (Research Experience for Undergraduates) funded by a grant from the National Science Foundation (Award \# 1560401). We, the "Solid Mechanics Team" of 2019, investigated a paper by Konda \& Erdogan 1994 (Konda \& Erdogan, 1994) in which a mixed mode crack problem has been solved, and we have filled in every theoretical step with explicit mathematical details. It is highly recommended that the reader analyze the paper (Konda \& Erdogan, 1994) while reading this one.

\subsection{Steps of the Singular Integral Equation Method}

The formulation of the crack problem follows six uniform steps (Chan, Paulino, \& Fannjiang, 2001; Erdogan, 1978; Erdogan \& Ozturk 1992; Muskhelishvili, 1953; Sneddon, 1966; Sneddon \& Lowengrub, 1969):

1. Linear elasticity and geometry of the crack problem

2. Governing partial differential equation (PDE) system

3. Implementing the Fourier transform and the inverse Fourier transform

4. Solving the ordinary differential equation system

5. Imposing boundary conditions

6. System of Fredholm integral equations

These steps make up the standard solution technique to the PDEs that arise in linear elasticity fracture mechanics (LEFM). Utilizing this outline, we expanded upon Konda and Erdogan (Konda \& Erdogan, 1994), adding mathematical details regarding the formulation of the crack problem. Once the Fredholm integral equation is obtained, we can discretize to form the numerical portion of the SIEM.

\section{Formulation of the Crack Problem}

\subsection{Linear Elasticity and Geometry of the Crack Problem}

We consider a plane elasticity problem, as shown in Figure 1, of a medium with a finite crack along the $y=0$ plane and a shear modulus $\mu$ given by

$$
\mu\left(x_{1}\right)=\mu_{0} e^{\delta x_{1}}, \mu(x, y)=\mu_{0} e^{\beta x+\gamma y},
$$

where

$$
\beta=\delta \cos (\theta), \gamma=\delta \sin (\theta),
$$

in which $\mu_{0}$ and $\delta$ are material constants. We treat Poisson's ratio, $v$, as a constant because its effect on stress intensity factors is insignificant (Delale \& Erdogan, 1983; Erdogan, 1978; Erdogan, 1995; Erdogan \& Ozturk, 1992; Konda \& Erdogan, 1994). From the relations $\kappa=3-4 v$ for plane strain and $\kappa=\frac{3-v}{1+\nu}$ for plane stress, the following equation is expressed for the Lamé modulus for plane stress

$$
\lambda(x, y)=\frac{3-\kappa}{\kappa-1} \mu_{0} e^{\beta x+\gamma y} .
$$




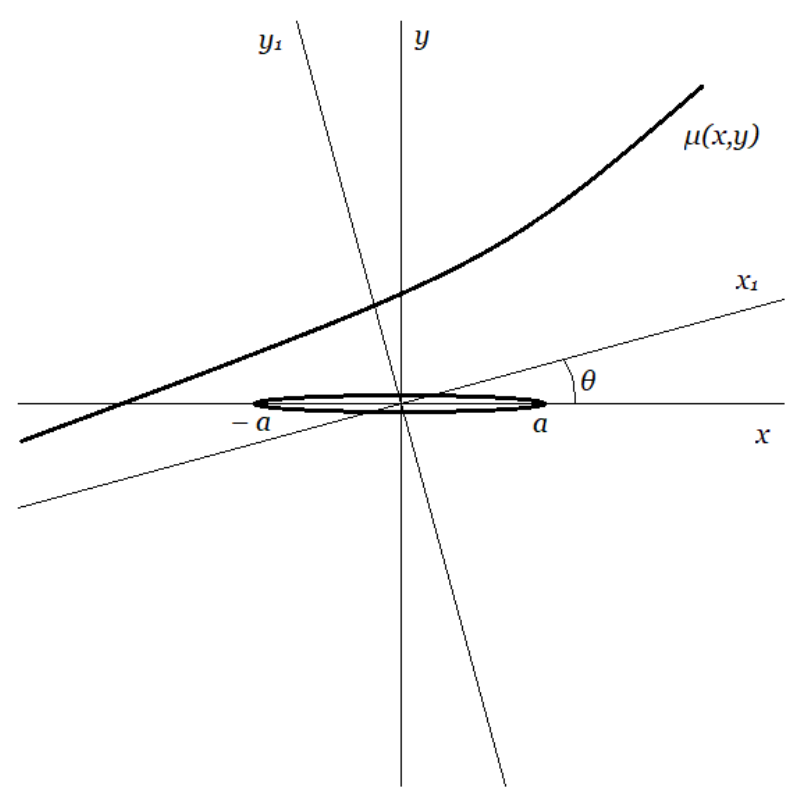

Figure 1. Crack geometry in the nonhomogeneous medium

\subsubsection{Displacement, Strain, and Stress Relations}

The relationship between the plane strain tensor $\varepsilon$ and displacement vector $\overrightarrow{\mathbf{u}}$ is given by $\varepsilon \equiv \frac{1}{2}\left(\nabla \overrightarrow{\mathbf{u}}+(\nabla \overrightarrow{\mathbf{u}})^{\top}\right)$. In two dimensions, this means that

$$
\varepsilon \equiv\left[\begin{array}{cc}
\frac{\partial u}{\partial x} & \frac{1}{2}\left(\frac{\partial u}{\partial y}+\frac{\partial v}{\partial x}\right) \\
\frac{1}{2}\left(\frac{\partial u}{\partial y}+\frac{\partial v}{\partial x}\right) & \frac{\partial v}{\partial y}
\end{array}\right],
$$

where $u$ and $v$ are $x$ - and $y$-components of $\overrightarrow{\mathbf{u}}$. Hooke's Law gives the following relation between strain and the stress tensor $\sigma$ :

$$
\sigma=2 \mu \varepsilon+\lambda \operatorname{tr}(\varepsilon) I,
$$

where $\mu$ and $\lambda$ are the shear modulus and the Lamé modulus, respectively, and $I$ is the identity matrix. Note that $\mu$ and $\lambda$ can, and in this paper will be, functions of position.

\subsection{2 (Generalized) Navier Equations}

For a material in equilibrium, we know that

$$
\nabla \cdot \sigma=0
$$

In terms of displacement, we use Hooke's Law and (6) to write this relationship as

$$
\nabla \cdot\left(2 \mu(x, y)\left[\begin{array}{cc}
\frac{\partial u}{\partial x} & \frac{1}{2}\left(\frac{\partial u}{\partial y}+\frac{\partial v}{\partial x}\right) \\
\frac{1}{2}\left(\frac{\partial u}{\partial y}+\frac{\partial v}{\partial x}\right) & \frac{\partial v}{\partial y}
\end{array}\right]+\lambda(x, y)\left[\begin{array}{cc}
\frac{\partial u}{\partial x}+\frac{\partial v}{\partial y} & 0 \\
0 & \frac{\partial u}{\partial x}+\frac{\partial v}{\partial y}
\end{array}\right]\right)=0
$$

Taking the divergence gives us the following system of PDEs:

$$
\begin{aligned}
& \frac{\partial \mu}{\partial x}\left(\frac{\partial u}{\partial x}+\frac{\partial u}{\partial x}\right)+\mu\left(\frac{\partial^{2} u}{\partial x^{2}}+\frac{\partial^{2} u}{\partial x^{2}}\right)+\frac{\partial \lambda}{\partial x}\left(\frac{\partial u}{\partial x}+\frac{\partial v}{\partial y}\right) \\
&+\lambda\left(\frac{\partial^{2} u}{\partial x^{2}}+\frac{\partial^{2} v}{\partial x \partial y}\right)+\frac{\partial \mu}{\partial y}\left(\frac{\partial u}{\partial y}+\frac{\partial v}{\partial x}\right)+\mu\left(\frac{\partial^{2} u}{\partial x^{2}}+\frac{\partial^{2} u}{\partial x \partial y}\right)=0 \\
& \frac{\partial \mu}{\partial x}\left(\frac{\partial v}{\partial x}+\frac{\partial u}{\partial y}\right)+\mu\left(\frac{\partial^{2} v}{\partial x^{2}}+\frac{\partial^{2} u}{\partial x \partial y}\right)+\frac{\partial \mu}{\partial y}\left(\frac{\partial v}{\partial y}+\frac{\partial v}{\partial y}\right) \\
&+\mu\left(\frac{\partial^{2} v}{\partial y^{2}}+\frac{\partial^{2} v}{\partial^{2} y}\right)+\frac{\partial \lambda}{\partial y}\left(\frac{\partial u}{\partial x}+\frac{\partial v}{\partial y}\right)+\lambda\left(\frac{\partial^{2} u}{\partial x \partial y}+\frac{\partial^{2} v}{\partial^{2} y}\right)=0
\end{aligned}
$$


For simplicity, we will only look at (10) and then generalize. First notice that we can group the $\mu$ terms as such:

$$
\mu\left(\frac{\partial^{2} u}{\partial x^{2}}+\frac{\partial^{2} u}{\partial y^{2}}\right)+\mu\left(\frac{\partial^{2} u}{\partial x^{2}}+\frac{\partial^{2} v}{\partial x \partial y}\right)=\mu \Delta u+\mu\left(\frac{\partial^{2} u}{\partial x^{2}}+\frac{\partial^{2} v}{\partial x \partial y}\right)
$$

where $\Delta$ represents the Laplacian operator. Next, we examine the partial derivatives of $\mu$ :

$$
\frac{\partial \mu}{\partial x}\left(\frac{\partial u}{\partial x}+\frac{\partial u}{\partial x}\right)+\frac{\partial \mu}{\partial y}\left(\frac{\partial u}{\partial y}+\frac{\partial v}{\partial x}\right)
$$

If we generalize this for both equations, we can see that the following relationship is a component of the Generalized Navier Equations:

$$
2 \varepsilon \nabla \mu=\left(\nabla \overrightarrow{\mathbf{u}}+(\nabla \overrightarrow{\mathbf{u}})^{\top}\right) \nabla \mu
$$

Now we look at partial derivatives of $\lambda$ :

$$
\frac{\partial \lambda}{\partial x}\left(\frac{\partial u}{\partial x}+\frac{\partial v}{\partial y}\right)+\frac{\partial \lambda}{\partial y}\left(\frac{\partial u}{\partial x}+\frac{\partial v}{\partial y}\right)=(\nabla \cdot \overrightarrow{\mathbf{u}}) \nabla \lambda .
$$

Finally, we take all of the remaining terms and combine them as such:

$$
\lambda\left(\frac{\partial^{2} u}{\partial x^{2}}+\frac{\partial^{2} v}{\partial x \partial y}\right)+\mu\left(\frac{\partial^{2} u}{\partial x^{2}}+\frac{\partial^{2} v}{\partial x \partial y}\right)=(\lambda+\mu)\left(\frac{\partial}{\partial x}(\nabla \cdot \overrightarrow{\mathbf{u}})\right) .
$$

Or in general, we get

$$
(\lambda+\mu) \nabla(\nabla \cdot \overrightarrow{\mathbf{u}})
$$

Combining (12), (14), (15), and (17), we get the full system of the Generalized Navier Equations:

$$
\mu \Delta \overrightarrow{\mathbf{u}}+(\lambda+\mu) \nabla(\nabla \cdot \overrightarrow{\mathbf{u}})+\left(\nabla \overrightarrow{\mathbf{u}}+\left(\nabla \overrightarrow{\mathbf{u}}^{\top}\right)\right) \nabla \mu+(\nabla \cdot \overrightarrow{\mathbf{u}}) \nabla \lambda=0,
$$

where $\mu, \lambda$, and $\overrightarrow{\mathbf{u}}$ are all functions of $x$ and $y$.

\subsection{Governing PDE System}

Substituting the definitions of $\mu$ and $\lambda$ and considering each component separately, we obtain the system of PDEs

$$
\begin{aligned}
& \mu_{0} e^{\beta x+\gamma y}\left[\frac{\partial^{2} u}{\partial x^{2}}+\frac{\partial^{2} u}{\partial y^{2}}+\left(\frac{3-\kappa}{\kappa-1}+1\right)\left(\frac{\partial^{2} u}{\partial x^{2}}+\frac{\partial v}{\partial x \partial y}\right)+2 \beta \frac{\partial u}{\partial x}+\gamma\left(\frac{\partial u}{\partial y}+\frac{\partial v}{\partial x}\right)+\beta\left(\frac{\partial u}{\partial x}+\frac{\partial v}{\partial y}\right)\left(\frac{3-\kappa}{\kappa-1}\right)\right]=0 \\
& \mu_{0} e^{\beta x+\gamma y}\left[\frac{\partial^{2} v}{\partial x^{2}}+\frac{\partial^{2} v}{\partial y^{2}}+\left(\frac{3-\kappa}{\kappa-1}+1\right)\left(\frac{\partial^{2} u}{\partial x \partial y}+\frac{\partial v}{\partial y^{2}}\right)+2 \gamma \frac{\partial v}{\partial y}+\beta\left(\frac{\partial u}{\partial y}+\frac{\partial v}{\partial x}\right)+\gamma\left(\frac{\partial u}{\partial x}+\frac{\partial v}{\partial y}\right)\left(\frac{3-\kappa}{\kappa-1}\right)\right]=0
\end{aligned}
$$

By multiplying through by $\frac{\kappa-1}{\mu_{0} e^{\beta x+y y}}$, we get the following form:

$$
\begin{aligned}
& (\kappa+1) \frac{\partial^{2} u}{\partial x^{2}}+(\kappa-1) \frac{\partial^{2} u}{\partial y^{2}}+2 \frac{\partial^{2} v}{\partial x \partial y}+\beta(\kappa+1) \frac{\partial u}{\partial x}+\gamma(\kappa-1) \frac{\partial u}{\partial y}+\gamma(\kappa-1) \frac{\partial v}{\partial x}+\beta(3-\kappa) \frac{\partial v}{\partial y}=0 \\
& (\kappa-1) \frac{\partial^{2} v}{\partial x^{2}}+(\kappa+1) \frac{\partial^{2} v}{\partial y^{2}}+2 \frac{\partial^{2} u}{\partial x \partial y}+\gamma(3-\kappa) \frac{\partial u}{\partial x}+\beta(\kappa-1) \frac{\partial u}{\partial y}+\beta(\kappa-1) \frac{\partial v}{\partial x}+\gamma(\kappa+1) \frac{\partial v}{\partial y}=0
\end{aligned}
$$

where $u$ and $v$ represent the $x$ and $y$ components of the displacement vector, respectively. When the solution of the uncracked material subjected to the given conditions and external loads, the problem would be solved by placing the following boundary conditions and self-equilibrating crack surface traction $p_{1}$ and $p_{2}$ :

$$
\begin{array}{rlrl}
\sigma_{y y}(x,+0)=\sigma_{y y}(x,-0), & \sigma_{x y}(x,+0)=\sigma_{x y}(x,-0) \\
v(x,+0)-v(x,-0) & =0, & & |x|>a \\
\sigma_{y y}(x,+0) & =p_{1}(x), & |x|<a \\
& & \\
u(x,+0)-u(x,-0) & =0, & & |x|>a \\
\sigma_{x y}(x,+0) & =p_{2}(x), & |x|<a .
\end{array}
$$




\subsection{Fourier Transform}

The Fourier transform is a common tool that can transform a linear PDE system into an ODE system, which can then be solved using elementary methods (Chan \& Koshkin, 2019). For the purposes of this paper, we will define the Fourier transform and inverse Fourier transform, respectively, as

$$
\begin{aligned}
(\mathcal{F} f)(\alpha) & \equiv \int_{-\infty}^{\infty} f(x) e^{i \alpha x} \mathrm{~d} x \equiv F(\alpha) \\
\left(\mathcal{F}^{-1} F\right)(x) & \equiv \frac{1}{2 \pi} \int_{-\infty}^{\infty} F(\alpha) e^{-i \alpha x} \mathrm{~d} \alpha \equiv f(x) .
\end{aligned}
$$

We can now express $u$ and $v$ as inverse Fourier transforms of two functions $U$ and $V$, i.e.

$$
\begin{aligned}
& u(x, y)=\frac{1}{2 \pi} \int_{-\infty}^{\infty} U(y, \alpha) e^{-i x \alpha} d \alpha \\
& v(x, y)=\frac{1}{2 \pi} \int_{-\infty}^{\infty} V(y, \alpha) e^{-i x \alpha} d \alpha,
\end{aligned}
$$

and then substitute these expressions for $u$ and $v$ into the system. Note that because of differentiation under the integral sign when we substitute, each partial differentiation by $x$ corresponds to multiplying the term by $-i \alpha$, while each partial differentiation by $y$ will still be a partial differentiation by $y$. The system then becomes

$$
\begin{aligned}
& (\kappa+1)\left(-\alpha^{2}\right) U+(\kappa-1) \frac{\partial^{2} U}{\partial y^{2}}+2(-i \alpha) \frac{\partial V}{\partial y}+\beta(\kappa+1)(-i \alpha) U+\gamma(\kappa-1) \frac{\partial U}{\partial y}+\gamma(\kappa-1)(-i \alpha) V+\beta(3-\kappa) \frac{\partial V}{\partial y}=0 \\
& (\kappa-1)\left(-\alpha^{2}\right) V+(\kappa+1) \frac{\partial^{2} V}{\partial y^{2}}+2(-i \alpha) \frac{\partial U}{\partial y}+\gamma(3-\kappa)(-i \alpha) U+\beta(\kappa-1) \frac{\partial U}{\partial y}+\beta(\kappa-1)(-i \alpha) V+\gamma(\kappa+1) \frac{\partial V}{\partial y}=0 .
\end{aligned}
$$

We now have partial derivatives only with respect to $y$, so we can treat $\alpha$ as a constant and solve the system as an ODE system with respect to $y$.

\subsection{Solution to the ODE System}

Isolating the second partials in these equations, we have four equations which we can express as a linear system with matrix notation:

$$
\left[\begin{array}{c}
U \\
U^{\prime} \\
V \\
V^{\prime}
\end{array}\right]^{\prime}=\left[\begin{array}{cccc}
0 & 1 & 0 & 0 \\
\frac{(\kappa+1)\left(\alpha^{2}+i \alpha \beta\right)}{\kappa-1} & -\gamma & i \alpha \gamma & \frac{\beta(\kappa-3)-2 i \alpha}{\kappa-1} \\
0 & 0 & 0 & 1 \\
\frac{\gamma(3-\kappa) i \alpha}{\kappa+1} & \frac{2 i \alpha-\beta(\kappa-1)}{\kappa+1} & \frac{(\kappa-1)\left(\alpha^{2}+i \alpha \beta\right)}{\kappa+1} & -\gamma
\end{array}\right]\left[\begin{array}{c}
U \\
U^{\prime} \\
V \\
V^{\prime}
\end{array}\right] .
$$

The solution to this system is the superposition of $e^{n_{j} y}, j \in\{1, \ldots, 4\}$, where $n_{j}$ are the eigenvalues of the $4 \times 4$ matrix in equation (31). The characteristic equation of the matrix is

$$
n^{4}+2 n^{3} \gamma+\left[-2 \alpha(\alpha+i \beta)+\gamma^{2}+\beta^{2} \frac{\kappa-3}{\kappa+1}\right] n^{2}+\alpha \gamma\left(-2 \alpha-i \beta \frac{8}{\kappa+1}\right) n+\alpha^{2}\left(\alpha^{2}+2 i \alpha \beta-\beta^{2}+\gamma^{2} \frac{3-\kappa}{\kappa+1}\right)=0,
$$

which can be rewritten as (Konda \& Erdogan, 1994)

$$
\left[n^{2}+n \gamma-\alpha(\alpha+i \beta)\right]^{2}+\frac{3-\kappa}{\kappa+1}(\alpha \gamma-i n \beta)^{2}=0 .
$$

Then, moving the second term over to the right side, taking the square root on both sides, and applying the quadratic formula, we find that

$$
\begin{array}{ll}
n_{1}=\frac{-\Delta_{1}}{2}-\frac{\sqrt{\Delta_{1}^{2}+4\left(\alpha^{2}+i \alpha \Delta_{2}\right)}}{2}, & n_{2}=\frac{-\Delta_{3}}{2}-\frac{\sqrt{\Delta_{3}^{2}+4\left(\alpha^{2}+i \alpha \Delta_{4}\right)}}{2}, \\
n_{3}=\frac{-\Delta_{1}}{2}+\frac{\sqrt{\Delta_{1}^{2}+4\left(\alpha^{2}+i \alpha \Delta_{2}\right)}}{2}, & n_{4}=\frac{-\Delta_{3}}{2}+\frac{\sqrt{\Delta_{3}^{2}+4\left(\alpha^{2}+i \alpha \Delta_{4}\right)}}{2},
\end{array}
$$

where

$$
\Delta_{1}=\sqrt{\frac{3-\kappa}{\kappa+1}} \beta+\gamma, \quad \Delta_{2}=\beta-\sqrt{\frac{3-\kappa}{\kappa+1}} \gamma, \quad \Delta_{3}=-\sqrt{\frac{3-\kappa}{\kappa+1}} \beta+\gamma, \quad \Delta_{4}=\beta+\sqrt{\frac{3-\kappa}{\kappa+1}} \gamma .
$$


Therefore, we could write the solutions as

$$
U(y, \alpha)=\sum_{j=1}^{4} G_{j}(\alpha) e^{y n_{j}}, \quad V(y, \alpha)=\sum_{j=1}^{4} F_{j}(\alpha) e^{y n_{j}},
$$

where $G_{j}$ and $F_{j}$ are unknown functions. However, note that these expressions leave eight unknowns, whereas there only need to be four (Konda $\&$ Erdogan, 1994). We can let $m_{j}=\frac{G_{j}}{F_{j}}$, i.e.

$$
U(y, \alpha)=\sum_{j=1}^{4} m_{j} F_{j}(\alpha) e^{y n_{j}}, \quad V(y, \alpha)=\sum_{j=1}^{4} F_{j}(\alpha) e^{y n_{j}}
$$

Substituting (37) into (29) and solving for $m_{j}$, we find that, for $j \in\{1, \ldots, 4\}$,

$$
m_{j}=\frac{[i 2 \alpha-\beta(3-\kappa)] n_{j}+i(\kappa-1) \alpha \gamma}{(\kappa-1) n_{j}^{2}+(\kappa-1) \gamma n_{j}-(\kappa+1) \alpha(\alpha+i \beta)} .
$$

Our solution is now reduced to four unknowns.

\subsection{Imposition of Boundary Conditions}

Physical conditions require that $u$ and $v$ must vanish for $x^{2}+y^{2} \rightarrow \infty$ (Konda \& Erdogan 1994). Based on (34), $n_{1}$ and $n_{2}$ have negative real parts while $n_{3}$ and $n_{4}$ have positive real parts. Therefore, to make the displacements approach zero in each half-plane, (37) requires

$$
\begin{gathered}
F_{3}(\alpha)=F_{4}(\alpha)=0, y>0 \\
F_{1}(\alpha)=F_{2}(\alpha)=0, y<0 .
\end{gathered}
$$

By using Hooke's Law, from (28) and (39) we obtain

$$
\begin{gathered}
\sigma_{x x}(x, y)=\frac{1}{2 \pi} \int_{-\infty}^{\infty} \sum_{j=1}^{l+1}\left[-i m_{j} \alpha(2 \mu+\lambda)+n_{j} \lambda\right] F_{j} e^{y n_{j}} e^{-i x \alpha} d \alpha \\
\sigma_{y y}(x, y)=\frac{1}{2 \pi} \int_{-\infty}^{\infty} \sum_{j=1}^{l+1}\left[-i m_{j} \alpha \lambda+n_{j}(2 \mu+\lambda)\right] F_{j} e^{y n_{j}} e^{-i x \alpha} d \alpha \\
\sigma_{x y}(x, y)=\frac{1}{2 \pi} \int_{-\infty}^{\infty} \sum_{j=1}^{l+1}\left[n_{j} m_{j}-i \alpha\right] F_{j} e^{y n_{j}} e^{-i x \alpha} d \alpha
\end{gathered}
$$

where $l=1$ for $y>0$ and $l=3$ for $y<0$. We can see that

$$
\sigma_{y y}\left(x, 0^{+}\right)=\frac{1}{2 \pi} \int_{-\infty}^{\infty}\left[\left(-i \alpha m_{1} \lambda+n_{1}(2 \mu+\lambda)\right) F_{1}+\left(-i \alpha m_{2} \lambda+n_{2}(2 \mu+\lambda)\right) F_{2}\right] e^{-i \alpha x} \mathrm{~d} \alpha
$$

and

$$
\sigma_{y y}\left(x, 0^{-}\right)=\frac{1}{2 \pi} \int_{-\infty}^{\infty}\left[\left(-i \alpha m_{3} \lambda+n_{3}(2 \mu+\lambda)\right) F_{3}+\left(-i \alpha m_{4} \lambda+n_{4}(2 \mu+\lambda)\right) F_{4}\right] e^{-i \alpha x} \mathrm{~d} \alpha .
$$

If we now substitute (41) into the homogeneous conditions (23) and substitute the definitions of $\lambda$ and $\mu$, we get

$$
c_{1} F_{1}+c_{2} F_{2}=c_{3} F_{3}+c_{4} F_{4}
$$

where

$$
\begin{aligned}
& c_{1}=-i \alpha m_{1}(3-\kappa)+n_{1}(\kappa-1) ; \\
& c_{2}=-i \alpha m_{2}(3-\kappa)+n_{2}(\kappa+1) \\
& c_{3}=-i \alpha m_{3}(3-\kappa)+n_{3}(\kappa-1) ; \\
& c_{4}=-i \alpha m_{4}(3-\kappa)+n_{4}(\kappa+1) .
\end{aligned}
$$


Similarly, we find that (42) substituted into (23) gives us

$$
d_{1} F_{1}+d_{2} F_{2}=d_{3} F_{3}+d_{4} F_{4}
$$

where

$$
\begin{aligned}
& d_{1}=n_{1} m_{1}-i \alpha ; \\
& d_{2}=n_{2} m_{2}-i \alpha ; \\
& d_{3}=n_{3} m_{3}-i \alpha ; \\
& d_{4}=n_{4} m_{4}-i \alpha .
\end{aligned}
$$

This gives us a system of equations that takes the form

$$
\left[\begin{array}{ll}
c_{3} & c_{4} \\
d_{3} & d_{4}
\end{array}\right]\left[\begin{array}{l}
F_{3} \\
F_{4}
\end{array}\right]=\left[\begin{array}{l}
c_{1} F_{1}+c_{2} F_{2} \\
d_{1} F_{1}+d_{2} F_{2}
\end{array}\right] .
$$

Solving the system in (55) gives us

$$
F_{3}=R_{1} F_{1}+R_{2} F_{2}, F_{4}=R_{3} F_{1}+R_{4} F_{2},
$$

where the known functions $R_{1}(\alpha), \ldots, R_{4}(\alpha)$ are given by

$$
\begin{gathered}
R_{1}(\alpha)=\left\{\left(m_{4}-m_{1}\right)\left[(1+\kappa) n_{1} n_{4}+(3-\kappa) \alpha^{2}\right]+i \alpha\left(n_{4}-n_{1}\right)\left[1+\kappa-(3-\kappa) m_{1} m_{4}\right]\right\} / R_{0} \\
R_{2}(\alpha)=\left\{\left(m_{4}-m_{2}\right)\left[(1+\kappa) n_{2} n_{4}+(3-\kappa) \alpha^{2}\right]+i \alpha\left(n_{4}-n_{2}\right)\left[1+\kappa-(3-\kappa) m_{2} m_{4}\right]\right\} / R_{0} \\
R_{3}(\alpha)=-\left\{\left(m_{3}-m_{1}\right)\left[(1+\kappa) n_{1} n_{3}+(3-\kappa) \alpha^{2}\right]+i \alpha\left(n_{3}-n_{1}\right)\left[1+\kappa-(3-\kappa) m_{1} m_{3}\right]\right\} / R_{0} \\
R_{4}(\alpha)=-\left\{\left(m_{3}-m_{2}\right)\left[(1+\kappa) n_{2} n_{3}+(3-\kappa) \alpha^{2}\right]+i \alpha\left(n_{3}-n_{2}\right)\left[1+\kappa-(3-\kappa) m_{2} m_{3}\right]\right\} / R_{0} \\
R_{0}(\alpha)=-\left\{\left(m_{4}-m_{3}\right)\left[(1+\kappa) n_{3} n_{4}+(3-\kappa) \alpha^{2}\right]+i \alpha\left(n_{4}-n_{3}\right)\left[1+\kappa-(3-\kappa) m_{3} m_{4}\right]\right\}
\end{gathered}
$$

\subsection{Fredholm Integral Equations}

To set up the system of integral equations, we introduce the following unknown functions:

$$
\begin{aligned}
& g_{1}(x) \equiv \frac{\partial}{\partial x}[u(x,+0)-u(x,-0)],|x|<a \\
& g_{2}(x) \equiv \frac{\partial}{\partial x}[v(x,+0)-v(x,-0)],|x|<a .
\end{aligned}
$$

These unknown functions are the strain at the crack surface, and since strain is the derivative of displacement, inserting them into our integral equations will give us convenient expressions for displacement. Now by plugging in the formulas for $u$ and $v$ given in (28) and then performing the Fourier transform on both sides, we obtain

$$
\begin{aligned}
\int_{-\infty}^{\infty} g_{1}(t) e^{i \alpha t} \mathrm{~d} t & =\int_{-\infty}^{\infty} e^{i \alpha t} \frac{\partial}{\partial t}[u(t,+0)-u(t,-0)] \mathrm{d} t \\
& =\int_{-\infty}^{\infty} e^{i \alpha t} \frac{\partial}{\partial t}\left(\frac{1}{2 \pi} \int_{-\infty}^{\infty}[U(+0, \alpha)-U(-0, \alpha)] e^{-i \alpha t} \mathrm{~d} \alpha\right) \mathrm{d} t \\
& =\int_{-\infty}^{\infty} e^{i \alpha t}\left(\frac{1}{2 \pi} \int_{-\infty}^{\infty}(-i \alpha)[U(+0, \alpha)-U(-0, \alpha)] e^{-i \alpha t} \mathrm{~d} \alpha\right) \mathrm{d} t \\
& =\mathcal{F F}^{-1}(-i \alpha[U(+0, \alpha)-U(-0, \alpha)]) \\
& =-i \alpha(U(+0, \alpha)-U(-0, \alpha)) .
\end{aligned}
$$

Using equations (37) and (39), the above equation becomes

$$
\int_{-\infty}^{\infty} g_{1}(t) e^{i \alpha t} \mathrm{~d} t=-i \alpha\left(m_{1} F_{1}+m_{2} F_{2}-m_{3} F_{3}-m_{4} F_{4}\right)=-i \alpha\left[\left(m_{1}-m_{3} R_{1}-m_{4} R_{3}\right) F_{1}+\left(m_{2}-m_{3} R_{2}-m_{4} R_{4}\right) F_{2}\right] .
$$

Similarly,

$$
\int_{-\infty}^{\infty} g_{2}(t) e^{i \alpha t} \mathrm{~d} t=-i \alpha\left[\left(1-R_{1}-R_{3}\right) F_{1}+\left(1-R_{2}-R_{4}\right) F_{2}\right]
$$


If we define

$$
\begin{aligned}
& f_{1 j} \equiv n_{3} m_{4} m_{j}\left(n_{4}-n_{j}\right)+n_{4} m_{3} m_{j}\left(n_{j}-n_{3}\right)+n_{j} m_{3} m_{4}\left(n_{3}-n_{4}\right) \\
& f_{2 j} \equiv m_{4} m_{j}\left(n_{4}-n_{j}\right)+m_{3} m_{j}\left(n_{j}-n_{3}\right)+m_{3} m_{4}\left(n_{3}-n_{4}\right) \\
& f_{3 j} \equiv n_{3} m_{3}\left(n_{4}-n_{j}\right)+n_{4} m_{4}\left(n_{j}-n_{3}\right)+n_{j} m_{j}\left(n_{3}-n_{4}\right) \\
& f_{4 j} \equiv m_{3}\left(n_{4}-n_{j}\right)+m_{4}\left(n_{j}-n_{3}\right)+m_{j}\left(n_{3}-n_{4}\right),
\end{aligned}
$$

and then let

$$
\begin{aligned}
a & \equiv \frac{-i \alpha(1+\kappa)}{R_{0}}\left(f_{11}-i \alpha f_{41}\right) \\
b & \equiv \frac{-i \alpha(1+\kappa)}{R_{0}}\left(f_{12}-i \alpha f_{42}\right) \\
c & \equiv \frac{-i \alpha}{R_{0}}\left(-(1+\kappa) f_{31}+i \alpha(3-\kappa) f_{21}\right) \\
d & \equiv \frac{-i \alpha}{R_{0}}\left(-(1+\kappa) f_{32}+i \alpha(3-\kappa) f_{22}\right),
\end{aligned}
$$

then we can factor $(65)$ and (66) as

$$
\begin{aligned}
& \int_{-\infty}^{\infty} g_{1}(t) e^{i \alpha t} \mathrm{~d} t=a F_{1}+b F_{2} \\
& \int_{-\infty}^{\infty} g_{2}(t) e^{i \alpha t} \mathrm{~d} t=c F_{1}+d F_{2} .
\end{aligned}
$$

We can then solve the system

to get

$$
\left[\begin{array}{ll}
a & b \\
c & d
\end{array}\right]\left[\begin{array}{l}
F_{1} \\
F_{2}
\end{array}\right]=\left[\begin{array}{l}
\int_{-\infty}^{\infty} g_{1}(t) e^{i \alpha t} \mathrm{~d} t \\
\int_{-\infty}^{\infty} g_{2}(t) e^{i \alpha t} \mathrm{~d} t
\end{array}\right]
$$

$$
\begin{gathered}
F_{1}=\frac{d \int_{-\infty}^{\infty} g_{1}(t) e^{i \alpha t} \mathrm{~d} t-b \int_{-\infty}^{\infty} g_{2}(t) e^{i \alpha t} \mathrm{~d} t}{a d-b c} \\
F_{2}=\frac{a \int_{-\infty}^{\infty} g_{2}(t) e^{i \alpha t} \mathrm{~d} t-c \int_{-\infty}^{\infty} g_{1}(t) e^{i \alpha t} \mathrm{~d} t}{a d-b c} .
\end{gathered}
$$

We need another set of equations to find $g_{1}$ and $g_{2}$ in terms of known functions. Substituting the equations from Hooke's Law into the boundary conditions (24) and (25), we get

$$
\lim _{y \rightarrow 0^{+}} \frac{1}{2 \pi} \int_{-\infty}^{\infty} \sum_{j=1}^{2}\left[-i \alpha m_{j} \lambda+n_{j}(2 \mu+\lambda)\right] F_{j}(\alpha) e^{n_{j} y} e^{-i \alpha x} \mathrm{~d} \alpha=p_{1}(x), \quad|x|<a
$$

and

$$
\lim _{y \rightarrow 0^{+}} \frac{1}{2 \pi} \int_{-\infty}^{\infty} \sum_{j=1}^{2} \mu\left(n_{j} m_{j}-i \alpha\right) F_{j}(\alpha) e^{n_{j} y} e^{-i \alpha x} \mathrm{~d} \alpha=p_{2}(x), \quad|x|<a .
$$

If we expand equations (74) and (75), we can get

$$
\begin{aligned}
& \lim _{y \rightarrow 0^{+}} \int_{-\infty}^{\infty}\left[\xi_{1} F_{1}+\eta_{1} F_{2}\right] e^{-i \alpha x} \mathrm{~d} \alpha=\frac{\pi(\kappa+1)}{2 \mu_{0}} e^{-\beta x} p_{1}(x) \\
& \lim _{y \rightarrow 0^{+}} \int_{-\infty}^{\infty}\left[\xi_{2} F_{1}+\eta_{2} F_{2}\right] e^{-i \alpha x} \mathrm{~d} \alpha=\frac{\pi(\kappa+1)}{2 \mu_{0}} e^{-\beta x} p_{2}(x)
\end{aligned}
$$

where

$$
\begin{aligned}
\xi_{1} & \equiv \frac{\kappa+1}{4(\kappa-1)}\left(-i \alpha m_{1}(3-\kappa)+n_{1}(\kappa+1)\right) e^{n_{1} y} \\
\eta_{1} & \equiv \frac{\kappa+1}{4(\kappa-1)}\left(-i \alpha m_{2}(3-\kappa)+n_{2}(\kappa+1)\right) e^{n_{2} y} \\
\xi_{2} & \equiv \frac{\kappa+1}{4}\left(n_{1} m_{1}-i \alpha\right) e^{n_{1} y} \\
\eta_{2} & \equiv \frac{\kappa+1}{4}\left(n_{2} m_{2}-i \alpha\right) e^{n_{2} y} .
\end{aligned}
$$


For equation (76) (and similarly for equation (77)), the integral on the left hand side becomes

$$
\int_{-\infty}^{\infty}\left[\left(\frac{\xi_{1} d-\eta_{1} c}{a d-b c}\right) \int_{-\infty}^{\infty} g_{1}(t) e^{i \alpha t} \mathrm{~d} t+\left(\frac{\eta_{1} a-\xi_{1} b}{a d-b c}\right) \int_{-\infty}^{\infty} g_{2}(t) e^{i \alpha t} \mathrm{~d} t\right] e^{-i \alpha x} \mathrm{~d} \alpha .
$$

We can group terms and note that displacement vanishes outside of the domain of the crack to rewrite (79) as

$$
\int_{-\infty}^{\infty}\left[K_{11}(y, \alpha) \int_{-a}^{a} g_{1}(t) e^{i \alpha t} \mathrm{~d} t+K_{12}(y, \alpha) \int_{-a}^{a} g_{2}(t) e^{i \alpha t} \mathrm{~d} t\right] e^{-i \alpha x} \mathrm{~d} \alpha
$$

where $K_{i j}$ is part of the Fredholm kernel, ${ }^{1}$ given by

$$
\begin{aligned}
K_{i 1} & =\frac{\xi_{i} d-\eta_{i} c}{a d-b c} \\
K_{i 2} & =\frac{\eta_{i} a-\xi_{i} b}{a d-b c} .
\end{aligned}
$$

Because $g_{1}$ and $g_{2}$ are only functions of $t$, we can switch the order of integration to get

$$
\int_{-a}^{a} h_{11}(x, y, t) g_{1}(t)+h_{12}(x, y, t) g_{2}(t) \mathrm{d} t,
$$

so in general we find that, for $|x|<a, k \in\{1,2\}$,

$$
\lim _{y \rightarrow 0^{+}} \int_{-a}^{a} \sum_{j=1}^{2} h_{k j}(x, y, t) g_{j}(t) \mathrm{d} t=\frac{\pi(\kappa+1)}{2 \mu_{0}} e^{-\beta x} p_{k}(x),
$$

where we define

$$
h_{k j} \equiv \int_{-\infty}^{\infty} K_{k j}(y, \alpha) e^{i \alpha(t-x)} \mathrm{d} \alpha, \quad k, j \in\{1,2\} .
$$

Since the difference in displacements must be zero at the crack tips, the system must be solved under the single-valuedness condition:

$$
\int_{-a}^{a} g_{1}(t) \mathrm{d} t=\int_{-a}^{a} g_{2}(t) \mathrm{d} t=0
$$

\subsubsection{Asymptotic Analysis of Kernels}

Some asymptotic analysis of the kernels as $|\alpha| \rightarrow \infty$ and $y \rightarrow 0$ is necessary in order to properly handle the improper integrals in (85), since integrating a non-vanishing function over an infinite domain will be problematic. We temporarily ignore the exponential multipliers in the kernel functions because they will approach their limit much more rapidly than the rest of the expressions. We can observe that

$$
n_{1}, n_{2}, n_{3}, n_{4} \stackrel{|\alpha| \rightarrow \infty}{\longrightarrow} C_{1}-|\alpha|, C_{2}-|\alpha|, C_{3}+|\alpha|, C_{4}+|\alpha|,
$$

where $C_{1} \ldots C_{4}$ are constants. Furthermore, from (38),

$$
\begin{aligned}
m_{j} & \stackrel{|\alpha| \rightarrow \infty}{\longrightarrow} \frac{2 i \alpha n_{j}}{(\kappa-1) n_{j}^{2}-(\kappa+1) \alpha^{2}} \\
& \rightarrow \pm \frac{2 i \alpha(-|\alpha|)+O(\alpha)}{-2 \alpha^{2}+O(\alpha)} \\
& \rightarrow \pm \frac{i|\alpha|}{\alpha},
\end{aligned}
$$

with a plus sign if $j=1,2$ and a minus sign otherwise. $(O(\alpha)$ here represents terms of degree less than 1 in $\alpha$, as in Big- $O$ notation.) The kernel expressions given in the appendix have a denominator with a factor of $\omega_{0}$ which is defined for the sake of concision. The equation for $\omega_{0}$ in the appendix is given as presented in Konda and Erdogan (Konda \& Erdogan, 1994). Note that we can re-factor $\omega_{0}$ the expression as

$$
\omega_{0}=\left(n_{1}-n_{3}\right)\left(n_{2}-n_{4}\right)\left(m_{1} m_{3}+m_{2} m_{4}\right)-\left(n_{1}-n_{2}\right)\left(n_{3}-n_{4}\right)\left(m_{1} m_{2}+m_{3} m_{4}\right)-\left(n_{1}-n_{4}\right)\left(n_{2}-n_{3}\right)\left(m_{1} m_{4}+m_{2} m_{3}\right) .
$$

\footnotetext{
${ }^{1}$ The expressions for these kernels are given in a more convenient form for the asymptotic analysis in the appendix.
} 
Since $m_{i}$ will converge more quickly than the $n_{i}$ for $i=1, \ldots, 4$, we can see that

$$
\begin{aligned}
\omega_{0} & \stackrel{|\alpha| \rightarrow \infty}{\longrightarrow} 2\left[\left(n_{1}-n_{3}\right)\left(n_{2}-n_{4}\right)+\left(n_{1}-n_{2}\right)\left(n_{3}-n_{4}\right)-\left(n_{1}-n_{4}\right)\left(n_{2}-n_{3}\right)\right] \\
& \stackrel{|\alpha| \rightarrow \infty}{\longrightarrow} 4\left(C_{1}-C_{2}\right)\left(C_{3}-C_{4}\right) .
\end{aligned}
$$

Similar re-factoring and analysis of the $f_{i j}$ in (67) will reveal that

$$
\begin{aligned}
& f_{1 j} \stackrel{|\alpha| \rightarrow \infty}{\longrightarrow}-2\left(C_{j}-|\alpha|\right)\left(C_{3}-C_{4}\right) \\
& f_{2 j} \stackrel{|\alpha| \rightarrow \infty}{\longrightarrow}-2\left(C_{3}-C_{4}\right) \\
& f_{3 j} \stackrel{|\alpha| \rightarrow \infty}{\longrightarrow} 2 i \frac{|\alpha|}{\alpha}\left(C_{j}-|\alpha|\right)\left(C_{3}-C_{4}\right) \\
& f_{4 j} \stackrel{|\alpha| \rightarrow \infty}{\longrightarrow} 2 i \frac{|\alpha|}{\alpha}\left(C_{3}-C_{4}\right) .
\end{aligned}
$$

Then, substituting into the kernel equations in the appendix, we can see that

$$
\begin{aligned}
& K_{11}(y, \alpha) \stackrel{|\alpha| \rightarrow \infty}{\longrightarrow} 0 \\
& K_{12}(y, \alpha) \stackrel{|\alpha| \rightarrow \infty}{\longrightarrow} \frac{1}{2 i} \frac{|\alpha|}{\alpha} e^{-|\alpha| y} \equiv K_{12}^{\infty}(0, \alpha) \\
& K_{21}(y, \alpha) \stackrel{|\alpha| \rightarrow \infty}{\longrightarrow} \frac{1}{2 i} \frac{|\alpha|}{\alpha} e^{-|\alpha| y} \equiv K_{21}^{\infty}(0, \alpha) \\
& K_{22}(y, \alpha) \stackrel{|\alpha| \rightarrow \infty}{\longrightarrow} 0 .
\end{aligned}
$$

\subsubsection{Final System of Integral Equations}

Because $K_{11}$ and $K_{22}$ vanish, they can be left as they are; however, $K_{12}$ and $K_{21}$ need to have the asymptotic value handled separately. We can show that

$$
\begin{aligned}
\lim _{y \rightarrow 0} \int_{-\infty}^{\infty} K_{12}^{\infty}(y, \alpha) e^{i \alpha(t-x)} \mathrm{d} \alpha=\lim _{y \rightarrow 0} \int_{-\infty}^{\infty} \frac{1}{2 i} \frac{|\alpha|}{\alpha} e^{-|\alpha| y} e^{i \alpha(t-x)} \mathrm{d} \alpha \\
=\lim _{y \rightarrow 0} \frac{1}{2 i} \int_{-\infty}^{\infty} \frac{|\alpha|}{\alpha} e^{|\alpha| y}(\cos (\alpha(t-x))+i \sin (\alpha(t-x))) \mathrm{d} \alpha \\
=\lim _{y \rightarrow 0} \frac{1}{2 i} \int_{-\infty}^{\infty} \frac{|\alpha|}{\alpha} e^{|\alpha| y} i \sin (\alpha(t-x)) \mathrm{d} \alpha \\
=\lim _{y \rightarrow 0} \frac{1}{2} \int_{-\infty}^{\infty} \frac{|\alpha|}{\alpha} e^{|\alpha| y} \sin (\alpha(t-x)) \mathrm{d} \alpha \\
=\lim _{y \rightarrow 0} \int_{0}^{\infty} e^{|\alpha| y} \sin (\alpha(t-x)) \mathrm{d} \alpha \\
=\lim _{y \rightarrow 0} \frac{t-x}{(t-x)^{2}+y^{2}}=\frac{1}{t-x} .
\end{aligned}
$$

Then we can rewrite (84) for $|x|<a$ as

$$
\begin{aligned}
& \int_{-a}^{a}\left[\frac{g_{2}(t)}{t-x}+k_{11}(x, t) g_{1}(t)+k_{12}(x, t) g_{2}(t)\right] \mathrm{d} t=\frac{\pi(1+\kappa)}{2 \mu(x, 0)} p_{1}(x), \\
& \int_{-a}^{a}\left[\frac{g_{1}(t)}{t-x}+k_{21}(x, t) g_{1}(t)+k_{22}(x, t) g_{2}(t)\right] \mathrm{d} t=\frac{\pi(1+\kappa)}{2 \mu(x, 0)} p_{2}(x),
\end{aligned}
$$

where

$$
\begin{aligned}
& k_{11}(x, t) \equiv h_{11}(x, 0, t) \\
& k_{12}(x, t) \equiv \int_{-\infty}^{\infty}\left(K_{12}(0, \alpha)-K_{12}^{\infty}(0, \alpha)\right) e^{i \alpha(t-x)} \mathrm{d} \alpha \\
& k_{21}(x, t) \equiv \int_{-\infty}^{\infty}\left(K_{21}(0, \alpha)-K_{21}^{\infty}(0, \alpha)\right) e^{i \alpha(t-x)} \mathrm{d} \alpha \\
& k_{22}(x, t) \equiv h_{22}(x, 0, t) .
\end{aligned}
$$


Now $k_{12}(x, t)$ and $k_{21}(x, t)$ can be rewritten to remove the imaginary part as such:

$$
\begin{aligned}
& k_{12}(x, t)=\int_{0}^{\infty}\left(K_{12}(0, \alpha) e^{i \alpha(t-x)}+K_{12}(0,-\alpha) e^{-i \alpha(t-x)}-\sin (\alpha(t-x))\right) \mathrm{d} \alpha \\
& k_{21}(x, t)=\int_{0}^{\infty}\left(K_{21}(0, \alpha) e^{i \alpha(t-x)}+K_{21}(0,-\alpha) e^{-i \alpha(t-x)}-\sin (\alpha(t-x))\right) \mathrm{d} \alpha .
\end{aligned}
$$

Note that the asymptotic value has now been separated, integrated separately, and added back into the integral equation.

\section{Numerical Part of SIEM}

\subsection{Numerical Procedures}

\subsubsection{Normalization}

We first normalize the equation by introducing the quantities

$$
\begin{array}{r}
s \equiv \frac{t}{a} ; \quad r \equiv \frac{x}{a} ; \quad f_{i}(s) \equiv g_{i}(t) ; \quad L_{i j}(r, s) \equiv k_{i j}(x, t) ; \\
p_{i}(a r)=p_{i}(x) ; \quad i, j \in\{1,2\} .
\end{array}
$$

The integral equations can then be rewritten as

$$
\begin{aligned}
& \frac{1}{\pi} \int_{-1}^{1}\left[\frac{f_{2}(s)}{s-r}+L_{11}(r, s) f_{1}(s)+L_{12}(r, s) f_{2}(s)\right] \mathrm{d} s=\frac{1+\kappa}{2 \mu(a r, 0)} p_{1}(a r), \\
& \frac{1}{\pi} \int_{-1}^{1}\left[\frac{f_{1}(s)}{s-r}+L_{21}(r, s) f_{1}(s)+L_{22}(r, s) f_{2}(s)\right] \mathrm{d} s=\frac{1+\kappa}{2 \mu(a r, 0)} p_{2}(a r)
\end{aligned}
$$

for $|r|<1$.

\subsubsection{Tchebyshev Polynomial Expansion}

It can be shown that the fundamental solution to the normalized integral equations is $\frac{1}{\sqrt{1-s^{2}}}$. Therefore, we can express $f_{1}$ and $f_{2}$ in terms of density functions

$$
f_{1}(s)=\frac{\Phi_{1}(s)}{\sqrt{1-s^{2}}} \quad \text { and } \quad f_{2}(s)=\frac{\Phi_{2}(s)}{\sqrt{1-s^{2}}} .
$$

$\Phi_{1}$ and $\Phi_{2}$ can be approximated by the Tchebyshev polynomial expansions

$$
\Phi_{1}(s)=\sum_{n=0}^{\infty} A_{n} T_{n}(s) \quad \text { and } \quad \Phi_{2}(s)=\sum_{n=0}^{\infty} B_{n} T_{n}(s),
$$

where $A_{n}$ and $B_{n}$ are constants and $T_{n}$ is the $n^{\text {th }}$ order Tchebyshev polynomial of the first kind. If we combine (110) and (111) with the single valuedness condition in (86) and note the orthogonality conditions of Tchebyshev polynomials, it follows that

$$
A_{0}=0 \quad \text { and } \quad B_{0}=0 .
$$

It is a well-known property that

$$
\frac{1}{\pi} \int_{-1}^{1} \frac{T_{n}(s)}{(s-r) \sqrt{1-s^{2}}} \mathrm{~d} s= \begin{cases}U_{n-1}(r) & |r|<1 \\ -\frac{\left(r-\frac{\mid r}{r} \sqrt{r^{2}-1}\right)^{n}}{\frac{|r|}{r} \sqrt{r^{2}-1}} & |r|>1\end{cases}
$$

where $U_{n}$ is the $n^{\text {th }}$ order Tchebyshev polynomial of the second kind (Konda $\&$ Erdogan, 1994). Using this property for $|r|<1$ and (112), we can rewrite the first terms in the integrals as

$$
\begin{aligned}
& \frac{1}{\pi} \int_{-1}^{1} \frac{f_{2}(s)}{s-r} \mathrm{~d} s=\sum_{n=1}^{\infty} B_{n} U_{n-1}(r) \\
& \frac{1}{\pi} \int_{-1}^{1} \frac{f_{1}(s)}{s-r} \mathrm{~d} s=\sum_{n=1}^{\infty} A_{n} U_{n-1}(r) .
\end{aligned}
$$


By substituting (110) and (111) into (109) and rewriting the first terms as above, we get

$$
\begin{array}{ll}
\sum_{n=1}^{\infty} B_{n} U_{n-1}(r)+\frac{1}{\pi} \sum_{n=1}^{\infty} \int_{-1}^{1} \frac{\left(A_{n} L_{11}(r, s)+B_{n} L_{12}(r, s)\right) T_{n}(s)}{\sqrt{1-s^{2}}} \mathrm{~d} s=\frac{(1+\kappa) p_{1}(a r)}{2 \mu(a r, 0)}, & |r|<1 ; \\
\sum_{n=1}^{\infty} A_{n} U_{n-1}(r)+\frac{1}{\pi} \sum_{n=1}^{\infty} \int_{-1}^{1} \frac{\left(A_{n} L_{21}(r, s)+B_{n} L_{22}(r, s)\right) T_{n}(s)}{\sqrt{1-s^{2}}} \mathrm{~d} s=\frac{(1+\kappa) p_{1}(a r)}{2 \mu(a r, 0)}, \quad|r|<1 .
\end{array}
$$

\subsubsection{Discretization}

We can approximate the left hand sides of (116) by truncating the series with a sufficiently high $N$. Furthermore, the remaining integral expressions can be turned into discretized sums via the collocation method. We want to discretize $r$ and $s$ in such a way that there are more points near the crack tips, so we define the discrete points as

$$
\begin{aligned}
s_{n} & =\cos \left(\frac{(2 n-1) \pi}{2(N+1)}\right), n=1,2, \ldots, N+1 \\
r_{n} & =\cos \left(\frac{n \pi}{N+1}\right), n=1,2, \ldots, N .
\end{aligned}
$$

Now we can use Gaussian quadrature to approximate the integrals as

$$
\frac{1}{\pi} \int_{-1}^{1} \frac{f(s)}{\sqrt{1-s^{2}}} \mathrm{~d} s \approx \frac{1}{N+1} \sum_{n=1}^{N+1} f\left(s_{n}\right),
$$

and, therefore, (116) can be written discretely as

$$
\begin{aligned}
& \left(U_{n-1}\left(r_{n}\right)+\sum_{i=1}^{N+1} \frac{L_{12}\left(r_{n}, s_{i}\right) T_{n}\left(s_{i}\right)}{N+1}\right) B_{n}+\sum_{i=1}^{N+1} \frac{L_{11}\left(r_{n}, s_{i}\right) T_{n}\left(s_{i}\right)}{N+1} A_{n}=\frac{1+\kappa}{2 \mu\left(a r_{n}, 0\right)} p_{1}\left(a r_{n}\right) \\
& \left(U_{n-1}\left(r_{n}\right)+\sum_{i=1}^{N+1} \frac{L_{21}\left(r_{n}, s_{i}\right) T_{n}\left(s_{i}\right)}{N+1}\right) A_{n}+\sum_{i=1}^{N+1} \frac{L_{22}\left(r_{n}, s_{i}\right) T_{n}\left(s_{i}\right)}{N+1} B_{n}=\frac{1+\kappa}{2 \mu\left(a r_{n}, 0\right)} p_{2}\left(a r_{n}\right)
\end{aligned}
$$

with $n=1,2, \ldots, N$. This system can be written in matrix form as

$$
\begin{aligned}
& \left(\mathbf{U}+\frac{\mathbf{L}_{\mathbf{1 2}} \mathbf{T}}{N+1}\right) \vec{B}+\frac{\mathbf{L}_{\mathbf{1 1}} \mathbf{T}}{N+1} \vec{A}=\overrightarrow{P_{1}} \\
& \left(\mathbf{U}+\frac{\mathbf{L}_{\mathbf{2 1}} \mathbf{T}}{N+1}\right) \vec{A}+\frac{\mathbf{L}_{\mathbf{2 2}} \mathbf{T}}{N+1} \vec{B}=\overrightarrow{P_{2}}
\end{aligned}
$$

where $\vec{A}$ and $\vec{B}$ are the vectors of unknown constants, and $\vec{P}_{j} \equiv \frac{1+\kappa}{2 \mu(a r, 0)} \vec{p}_{j}(a r)$, where the division of vectors is element-wise. $\mathbf{U}, \mathbf{L}_{\mathbf{i j}}$, and $\mathbf{T}$ are matrices that take the following forms:

$$
\begin{gathered}
\mathbf{U}=\left[\begin{array}{cccc}
U_{0}\left(r_{1}\right) & U_{1}\left(r_{1}\right) & \ldots & U_{N-1}\left(r_{1}\right) \\
U_{0}\left(r_{2}\right) & U_{1}\left(r_{2}\right) & \ldots & U_{N-1}\left(r_{2}\right) \\
\vdots & \vdots & \ddots & \vdots \\
U_{0}\left(r_{N}\right) & U_{1}\left(r_{N}\right) & \ldots & U_{N-1}\left(r_{N}\right)
\end{array}\right] \\
\mathbf{T}=\left[\begin{array}{cccc}
T_{1}\left(s_{1}\right) & T_{2}\left(s_{1}\right) & \ldots & T_{N}\left(s_{1}\right) \\
T_{1}\left(s_{2}\right) & T_{2}\left(s_{2}\right) & \ldots & T_{N}\left(s_{2}\right) \\
\vdots & \vdots & \ddots & \vdots \\
T_{1}\left(s_{N+1}\right) & T_{2}\left(s_{N+1}\right) & \ldots & T_{N}\left(s_{N+1}\right)
\end{array}\right] \\
\mathbf{L}_{\mathbf{i j}}=\left[\begin{array}{cccc}
L_{i j}\left(r_{1}, s_{1}\right) & L_{i j}\left(r_{1}, s_{2}\right) & \ldots & L_{i j}\left(r_{1}, s_{N+1}\right) \\
L_{i j}\left(r_{2}, s_{1}\right) & L_{i j}\left(r_{2}, s_{2}\right) & \ldots & L_{i j}\left(r_{2}, s_{N+1}\right) \\
\vdots & \vdots & \ddots & \vdots \\
L_{i j}\left(r_{N}, s_{1}\right) & L_{i j}\left(r_{N}, s_{2}\right) & \ldots & L_{i j}\left(r_{N}, s_{N+1}\right)
\end{array}\right]
\end{gathered}
$$


for $i, j \in\{1,2\}$. When we expand the equations of (122), we get two $N \times 2 N$ systems, which we can combine into a single $2 N \times 2 N$ matrix equation that takes the form of

$$
\left[\begin{array}{cccccc}
a_{11} & \ldots & a_{1 N} & b_{11} & \ldots & b_{1 N} \\
\vdots & \ddots & \ddots & \ddots & \ddots & \vdots \\
a_{N 1} & \ldots & a_{N N} & b_{N 1} & \ldots & b_{N N} \\
a_{(N+1) 1} & \ldots & a_{(N+1) N} & b_{(N+1) 1} & \ldots & b_{(N+1) N} \\
\vdots & \ddots & \ddots & \ddots & \ddots & \vdots \\
a_{(2 N) 1} & \ldots & a_{(2 N) N} & b_{(2 N) 1} & \ldots & b_{(2 N) N}
\end{array}\right]\left[\begin{array}{c}
A_{1} \\
\vdots \\
A_{N} \\
B_{1} \\
\vdots \\
B_{N}
\end{array}\right]=\left[\begin{array}{c}
p_{1} \\
\vdots \\
p_{1} \\
p_{2} \\
\vdots \\
p_{2}
\end{array}\right] .
$$

This system can easily be solved in MATLAB via matrix inversion to find each $A_{n}$ and $B_{n}$.

$$
\rightarrow \bar{k}_{1}(a) \longrightarrow \bar{k}_{1}(-a)--b \quad \bar{k}_{2}(a)--a-\bar{k}_{2}(-a)
$$

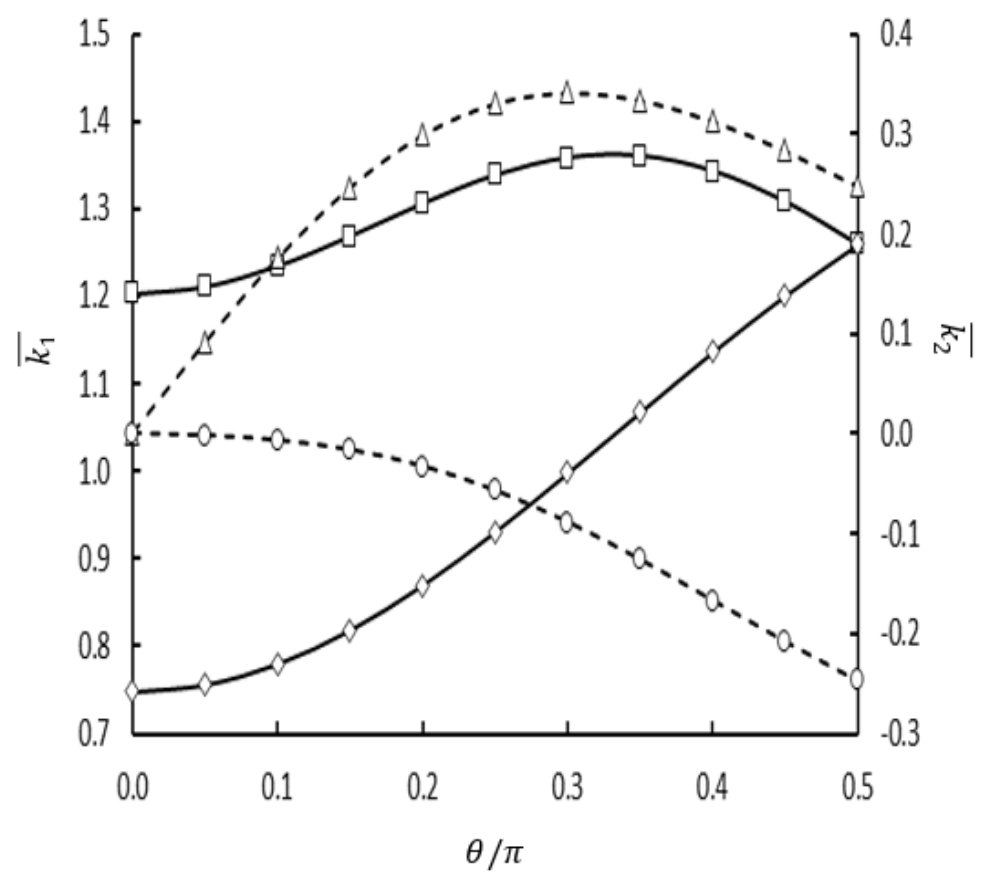

Figure 2. Variation of the normalized stress intensity factors with the crack orientation in a nonhomogeneous medium containing a uniformly pressurized crack, $a \delta=1$

3.1.4 Stress Intensity Factors (SIFs)

The stress intensity factors at the crack tips $a$ and $-a$ are given by (Konda \& Erdogan, 1994)

$$
\begin{aligned}
k_{1}(a) & =\lim _{x \rightarrow a^{+}} \sigma_{y y}(x, 0) \sqrt{2(x-a)}, \\
k_{1}(-a) & =\lim _{x \rightarrow-a^{-}} \sigma_{y y}(x, 0) \sqrt{2(-x-a)}, \\
k_{2}(a) & =\lim _{x \rightarrow a^{+}} \sigma_{x y}(x, 0) \sqrt{2(x-a)}, \\
k_{2}(-a) & =\lim _{x \rightarrow-a^{-}} \sigma_{x y}(x, 0) \sqrt{2(-x-a)} .
\end{aligned}
$$

To evaluate $k_{1}$ and $k_{2}$, we note that equations (84) and (101) give formulas for $\sigma_{y y}$ and $\sigma_{x y}$ that work for $|x|>a$ as well as 


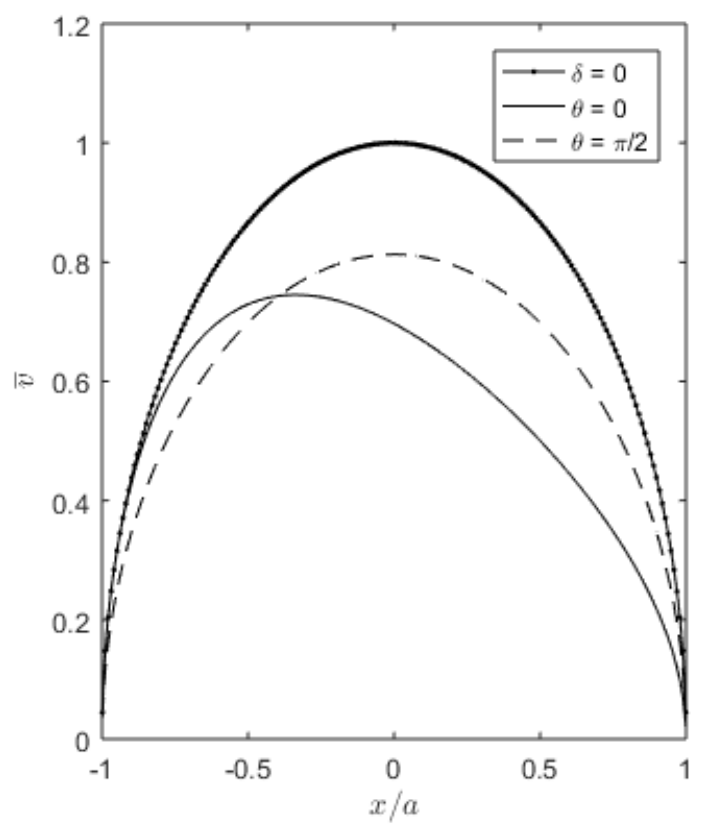

Figure 3. Normalized relative crack opening in the $y$ direction for $a \delta=0.5, \theta=0$, and $\theta=\pi / 2$ and for a homogeneous medium $(\delta=0)$

$|x|<a$. Therefore, we can use the property (113) and replace $p_{1}(x)$ with $\sigma_{y y}$ to show that

$$
\begin{aligned}
k_{1}(a) & =\lim _{x \rightarrow a^{+}} \sigma_{y y}(x, 0) \sqrt{2(x-a)} \\
& =\lim _{r \rightarrow 1^{+}} \sigma_{y y}(a r, 0) \sqrt{a} \sqrt{2(r-1)} \\
& =\lim _{r \rightarrow 1^{+}} \sqrt{a} \sqrt{2(r-1)} \frac{2 \mu(a r, 0)}{1+\kappa}\left[\sum_{n=1}^{\infty} B_{n}\left(-\frac{\left(r-\frac{|r|}{r} \sqrt{r^{2}-1}\right)^{n}}{\frac{|r|}{r} \sqrt{r^{2}-1}}\right)+F_{1}(r)\right]
\end{aligned}
$$

for $r>1$. Evaluating the limit, we find

$$
k_{1}(a)=-\sqrt{a} \frac{2 \mu(a, 0)}{1+\kappa} \sum_{n=1}^{\infty} B_{n} .
$$

Similarly, we can take limits and find

$$
\begin{aligned}
& k_{2}(a)=-\sqrt{a} \frac{2 \mu(a, 0)}{1+\kappa} \sum_{n=1}^{\infty} A_{n} ; \\
& k_{1}(-a)=-\sqrt{a} \frac{2 \mu(-a, 0)}{1+\kappa} \sum_{n=1}^{\infty}(-1)^{n} B_{n} ; \\
& k_{2}(-a)=-\sqrt{a} \frac{2 \mu(-a, 0)}{1+\kappa} \sum_{n=1}^{\infty}(-1)^{n} A_{n} .
\end{aligned}
$$

\subsection{Numerical Results}

In this section we display results normalized according to the formula

$$
\bar{k}_{i}(\mp a) \equiv \frac{k_{i}(\mp a)}{P \sqrt{a}}
$$

where $P$ is the amplitude of the crack surface traction. We calculated the SIFs as in equations (132)-(135) with $N=30$ collocation points, and these numbers are reported in Tables 1,2, and 3, which are analogous to the first two tables 


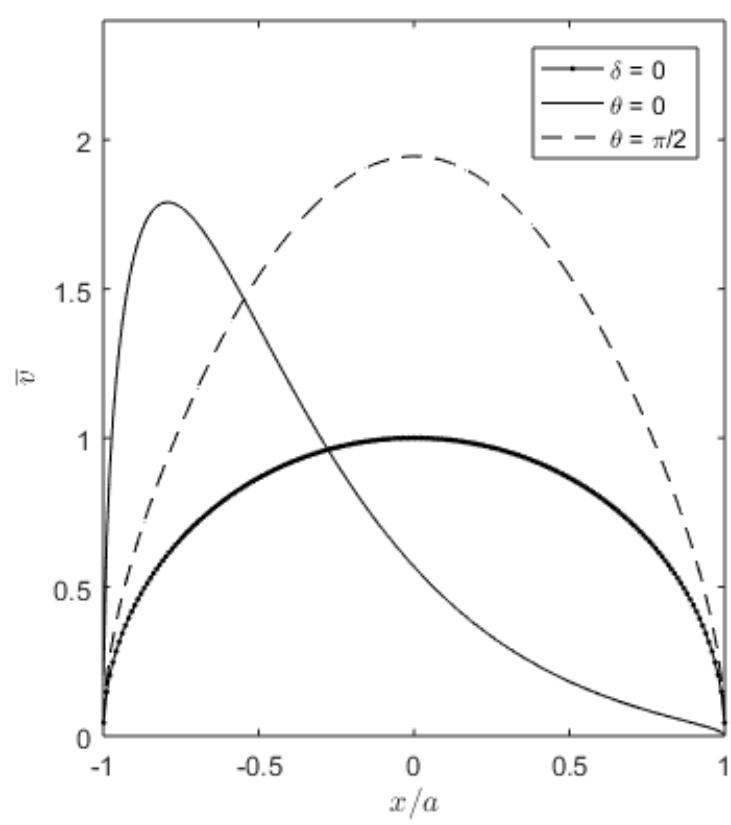

Figure 4. Normalized relative crack opening in the $y$ direction for $a \delta=2.5, \theta=0$, and $\theta=\pi / 2$ and for a homogeneous medium $(\delta=0)$

Table 1. The effect of the nonhomogeneity constant $\delta$ on the stress intensity factors; $v=0.3, p_{1}(x)=-\sigma_{0}, p_{2}(x)=0, \bar{k}_{i}(\mp a)=k_{i}(\mp a) / \sigma_{0} \sqrt{a}$

\begin{tabular}{cccccccc}
\hline \multicolumn{7}{c}{$a \delta$} \\
\hline$\theta / \pi$ & & 0.1 & 0.25 & 0.5 & 1.0 & 2.5 & 5.0 \\
\hline 0 & $\bar{k}_{1}(a)$ & 1.024 & 1.058 & 1.110 & 1.203 & 2.434 & \\
& $\bar{k}_{1}(-a)$ & 0.974 & 0.933 & 0.866 & 0.747 & 0.526 & \\
& $\bar{k}_{2}(a)$ & 0.000 & 0.000 & 0.000 & 0.000 & 0.000 & 0.000 \\
& $\bar{k}_{2}(-a)$ & 0000 & 0.000 & 0.000 & 0.000 & 0.000 & 0.000 \\
\hline 0.5 & $\bar{k}_{1}(a)$ & 1.009 & 1.037 & 1.101 & 1.259 & 1.801 & 2.752 \\
& $\bar{k}_{1}(-a)$ & 1.009 & 1.037 & 1.101 & 1.259 & 1.801 & 2.752 \\
& $\bar{k}_{2}(a)$ & 0.025 & 0.061 & 0.123 & 0.247 & 0.633 & 1.242 \\
& $\bar{k}_{2}(-a)$ & -0.025 & -0.061 & -0.123 & -0.247 & -0.633 & -1.242 \\
\hline
\end{tabular}

presented in (Konda \& Erdogan, 1994). Table 1 displays the effect of nonhomogeneity $(\delta)$ for a horizontal crack and one rotated $90^{\circ}$, and Tables 2 and 3 show the effect of both nonhomogeneity and crack orientation $(\theta)$ in more detail.

The results in Tables 2 and 3 are very similar to those presented by Konda and Erdogan (Konda \& Erdogan, 1994), with only a handful of values having any appreciable difference. This can be most clearly seen in Figure 2 where the values for $\bar{k}_{i}( \pm a)$ are plotted for $a \delta=1$ over all crack orientations in Tables 2 and 3. However, even these differences can be attributed to deviations in the specific numerical schemes, as computational techniques and technology have changed significantly since the original values were calculated.

After solving (101) for the unknown functions, $g_{1}$ and $g_{2}$, we can calculate the $x$ and $y$ displacements of the relative crack surface by using (62), (63), and the following relationship for $|r|<1$ :

$$
\int_{-1}^{r} \frac{T_{n}(s) \mathrm{d} s}{\sqrt{1-s^{2}}}=-\frac{1}{n} U_{n-1}(r) \sqrt{1-r^{2}}
$$

These are given by 
Table 2. The effects of the nonhomogeneity constant $(\delta)$ for crack orientation $\theta \in\{0, \ldots, 0.25 \pi\}$ on the SIFs; $v=0.3, p_{1}(x)=-\sigma_{0}, p_{2}(x)=0, \bar{k}_{i}(\mp a)=k_{i}(\mp a) / \sigma_{0} \sqrt{a}$

\begin{tabular}{|c|c|c|c|c|c|c|c|}
\hline \multicolumn{8}{|c|}{$\theta / \pi$} \\
\hline$a \delta$ & & 0 & 0.05 & 0.1 & 0.15 & 0.2 & 0.25 \\
\hline \multirow[t]{4}{*}{0.1} & $\bar{k}_{1}(a)$ & 1.024 & 1.024 & 1.024 & 1.024 & 1.023 & 1.022 \\
\hline & $\bar{k}_{1}(-a)$ & 0.974 & 0.974 & 0.976 & 0.978 & 0.982 & 0.986 \\
\hline & $\bar{k}_{2}(a)$ & 0.0000 & 0.0050 & 0.0097 & 0.0140 & 0.0176 & 0.0206 \\
\hline & $\bar{k}_{2}(-a)$ & 0.0000 & -0.0028 & -0.0056 & -0.0084 & -0.0113 & -0.0142 \\
\hline \multirow[t]{4}{*}{0.25} & $\bar{k}_{1}(a)$ & 1.058 & 1.059 & 1.060 & 1.062 & 1.063 & 1.064 \\
\hline & $\bar{k}_{1}(-a)$ & 0.933 & 0.934 & 0.940 & 0.948 & 0.959 & 0.972 \\
\hline & $\bar{k}_{2}(a)$ & 0.000 & 0.015 & 0.029 & 0.042 & 0.052 & 0.059 \\
\hline & $\bar{k}_{2}(-a)$ & 0.000 & -0.005 & -0.010 & -0.015 & -0.022 & -0.029 \\
\hline \multirow[t]{4}{*}{0.5} & $\bar{k}_{1}(a)$ & 1.110 & 1.113 & 1.119 & 1.129 & 1.138 & 1.146 \\
\hline & $\bar{k}_{1}(-a)$ & 0.866 & 0.870 & 0.882 & 0.902 & 0.927 & 0.956 \\
\hline & $\bar{k}_{2}(a)$ & 0.000 & 0.037 & 0.071 & 0.100 & 0.122 & 0.137 \\
\hline & $\bar{k}_{2}(-a)$ & 0.000 & -0.005 & -0.011 & -0.019 & -0.030 & -0.044 \\
\hline \multirow[t]{4}{*}{1.0} & $\bar{k}_{1}(a)$ & 1.203 & 1.211 & 1.235 & 1.269 & 1.306 & 1.338 \\
\hline & $\bar{k}_{1}(-a)$ & 0.747 & 0.756 & 0.780 & 0.818 & 0.870 & 0.931 \\
\hline & $\bar{k}_{2}(a)$ & 0.000 & 0.091 & 0.176 & 0.247 & 0.299 & 0.331 \\
\hline & $\bar{k}_{2}(-a)$ & 0.000 & -0.002 & -0.007 & -0.016 & -0.033 & -0.057 \\
\hline
\end{tabular}

Table 3. The effects of the nonhomogeneity constant $(\delta)$ for crack orientation $\theta \in\{0.3 \pi, \ldots, 0.5 \pi\}$ on the SIFs; $v=0.3, p_{1}(x)=-\sigma_{0}, p_{2}(x)=0, \bar{k}_{i}(\mp a)=k_{i}(\mp a) / \sigma_{0} \sqrt{a}$

\begin{tabular}{ccccccc}
\hline \multicolumn{7}{c}{$\theta / \pi$} \\
\hline$\delta \delta$ & & 0.3 & 0.35 & 0.4 & 0.45 & 0.5 \\
\hline 0.1 & $\bar{k}_{1}(a)$ & 1.021 & 1.018 & 1.016 & 1.012 & 1.0085 \\
& $\bar{k}_{1}(-a)$ & 0.990 & 0.995 & 1.000 & 1.004 & 1.0085 \\
& $\bar{k}_{2}(a)$ & 0.0228 & 0.0243 & 0.0251 & 0.0251 & 0.0245 \\
& $\bar{k}_{2}(-a)$ & -0.0169 & -0.0194 & -0.0216 & -0.0233 & -0.0245 \\
\hline 0.25 & $\bar{k}_{1}(a)$ & 1.063 & 1.059 & 1.054 & 1.046 & 1.037 \\
& $\bar{k}_{1}(-a)$ & 0.986 & 0.999 & 1.013 & 1.025 & 1.037 \\
& $\bar{k}_{2}(a)$ & 0.064 & 0.066 & 0.066 & 0.065 & 0.061 \\
& $\bar{k}_{2}(-a)$ & -0.036 & -0.044 & -0.050 & -0.056 & -0.061 \\
\hline 0.5 & $\bar{k}_{1}(a)$ & 1.149 & 1.146 & 1.137 & 1.122 & 1.101 \\
& $\bar{k}_{1}(-a)$ & 0.987 & 1.018 & 1.049 & 1.077 & 1.101 \\
& $\bar{k}_{2}(a)$ & 0.145 & 0.146 & 0.142 & 0.134 & 0.123 \\
& $\bar{k}_{2}(-a)$ & -0.059 & -0.076 & -0.093 & -0.109 & -0.123 \\
\hline 1.0 & $\bar{k}_{1}(a)$ & 1.358 & 1.360 & 1.343 & 1.308 & 1.259 \\
& $\bar{k}_{1}(-a)$ & 0.998 & 1.068 & 1.136 & 1.201 & 1.259 \\
& $\bar{k}_{2}(a)$ & 0.341 & 0.334 & 0.313 & 0.283 & 0.247 \\
& $\bar{k}_{2}(-a)$ & -0.089 & -0.126 & -0.167 & -0.208 & -0.247 \\
\hline
\end{tabular}

$$
\begin{aligned}
& u\left(x, 0^{+}\right)-u\left(x, 0^{-}\right)=\int_{-a}^{x} g_{1}(t) d t=-\sqrt{a^{2}-x^{2}} \sum_{1}^{\infty} \frac{1}{n} A_{n} U_{n-1}\left(\frac{x}{a}\right) \\
& v\left(x, 0^{+}\right)-v\left(x, 0^{-}\right)=\int_{-a}^{x} g_{2}(t) d t=-\sqrt{a^{2}-x^{2}} \sum_{1}^{\infty} \frac{1}{n} B_{n} U_{n-1}\left(\frac{x}{a}\right) .
\end{aligned}
$$

Figures 3 and 4 depict the normalized crack surface displacement in the $y$ direction $a \delta=0.5$ and $a \delta=2.5$ respectively, where the normalized displacement is given by

$$
\bar{v}=\frac{2 \mu_{0}}{\sigma_{0}(1+\kappa)}\left[v\left(x, 0^{+}\right)-v\left(x, 0^{-}\right)\right],
$$

and for a homogeneous medium, the displacement will just be a semicircle centered on the origin. In both cases, the magnitude of our calculated displacement curves is less than Konda and Erdogan's, but the shapes are consistent with our expectations. All curves have a steep slope at the crack tips, representing the strain going to infinity there. Additionally, 
the displacement of the crack surface for a highly nonhomogeneous medium $(a \delta=2.5)$ is still significantly greater than that of a more homogeneous medium $(a \delta=0.5)$.

\section{Concluding Remarks}

If readers place the paper by (Konda \& Erdogan, 1994) and ours side by side, it can be noticed that we have filled in almost every mathematical step from paper (Konda \& Erdogan, 1994) with comprehensive explanation and detailed mathematical derivation. Moreover, numerical computation is also broken down step by step with comprehensive remarks, and MATLAB programming files are accessible at github.com/SolidMechanicsREU2019/MixedModeCrack. The files are free to access, download, and easy to edit and revise for other types of crack problems.

In essence, our paper can be considered as a promotion for the singular integral equation method (SIEM). Due to the cracktip singularity, most numerical methods (such as finite element method or finite difference) are not suitable to solve crack problems. In contrast, SIEM is a unique and more accurate method for solving crack problems because of its capability of capturing the crack-tip singularity. Of course, as a general principle, there is a trade-off; the price we pay is the somewhat lengthy derivation that leads to the singular integral equation(s), and it also requires a few steps of analytical manipulation (i.e., exact evaluation of the singular integrals involving Tchebyshev polynomials) in the numerical part. Though many researchers have used SIEM to solve crack problems, those lengthy derivations and exact evaluations of singular integrals are not well documented or comprehensively explained. Here we present SIEM in a plain and organized way by breaking down the lengthy derivation step-by-step comprehensively by filling out the mathematical details. It is our hope that our work in this paper can be used as a standard "solution manual" for other researchers in fracture mechanics to solve crack problems.

\section{Acknowledgements}

Chan, Athaide, Belcher, and Kelly thank the grant (Award \# 1560401) from National Science Foundation REU (Research Experience for Undergraduates) program. Authors also like to thank University of Houston-Downtown (UHD) for the support and helps in summer 2019 for running the REU program.

\section{References}

Bhavar, V., Kattire, P., Thakare, S., \& Singh, R. K. P. (2017, September). A review on functionally gradient materials (FGMs) and their applications. In IOP Conference Series: Materials Science and Engineering (229(1), pp. 012021). IOP Publishing.

Chan, Y.-S., \& Koshkin, S. (2019). Mathematical details on singular integral equation method for solving crack problems. Journal of Mathematics Research, 11(1), 102-117. https://doi.org/10.5539/jmr.v11n1p102

Chan, Y.-S., Paulino, G. H., \& Fannjiang, A. C. (2001). The crack problem for nonhomogeneous materials under antiplane shear loading-a displacement based formulation. International Journal of Engineering Science, 38(17), 2989-3005.

Debnath, L. (1995). Integral Transforms and Their Applications. Boca Raton, Florida: CRC Press.

Delale, F., \& Erdogan, F. (1983). The crack problem for a nonhomogeneous plane. Journal of Applied Mechanics, Transactions ASME, 50(3), 609-614. https://doi.org/10.1115/1.3167098

Erdogan, F. (1978). Mixed boundary value problems in mechanics. In S. Nemat-Nasser, editor, Mechanics Today, 4, 1-86. New York, NY: Pergamon Press Inc. https://doi.org/10.1016/B978-0-08-021792-5.50009-4

Erdogan, F. (1995). Fracture mechanics of functionally graded materials. Composites Engineering, 5(7), 753-770. https://doi.org/10.1016/0961-9526(95)00029-M

Erdogan, F., \& Gupta, G. D. (1972). On the numerical solution of singular integral equations. Quarterly of Applied Mathematics, 30, 525-534. https://doi.org/10.1007/978-94-017-2260-5_7

Erdogan, F., Gupta, G. D., \& Cook, T. S. (1973). Numerical solution of singular integral equations. In Sih, G. C. (Ed.), Mechanics of Fracture, 1(7), 368-425. Leyden, The Netherlands: Noordhoff International Publishing. https://doi.org/10.1007/978-94-017-2260-5_7

Erdogan, F., \& Ozturk, M. (1992). Diffusion problems in bonded nonhomogeneous materials with an interface cut. International Journal of Engineering Science, 30(10), 1507-1523. https://doi.org/10.1016/0020-7225(92)90161-9

Evans, L. C. (1998). Partial Differential Equations. Providence, R.I.: American Mathematical Society.

Folland, G. B. (1992). Fourier Analysis and its Applications. Pacific Grove, California: Wadsworth \& Brooks/Cole Advanced Books \& Software.

Gu, P., \& Asaro, R. J. (1997). Cracks in functionally graded materials. International Journal of Scientific Study, 34(1), 


\section{1-17. https://doi.org/10.1016/0020-7683(95)00289-8}

Heer, B., \& Bandyopadhyay, A. (2018). Compositionally graded magnetic-nonmagnetic bimetallic structure using laser engineered net shaping. Materials Letters, 216, 16-19. https://doi.org/10.1016/j.matlet.2017.12.129

Hirai, T. (1996). Functionally gradient materials. In Brook, R.J. (Ed.), Materials Science and Technology, Processing of Ceramics, Part 2,17B, 292-341. Weinheim, Germany.

Jin, Z. H., \& Batra, R. C. (1996). Some basic fracture mechanics concepts in functionally graded materials. Journal of Mechanics and Physical Solids, 44(8), 1221-1235. https://doi.org/10.1016/0022-5096(96)00041-5

Jin, Z. H., \& Noda, N. (1994). Crack-tip singular fields in nonhomogeneous materials. Journal of Applied Mechanics, Transactions ASME, 61(3), 738-740. https://doi.org/10.1115/1.2901529

Kabir, H., Madenci, E., \& Ortega, A. (1998). Numerical solution of integral equations with logarithmic-, Cauchy- and Hadamard-type singularities. International Journal for Numerical Methods in Engineering, 41(4), 617-638.

Kawasaki, A., \& Watanabe, R. (1990). Fabrication of sintered functionally gradient materials by powder spray forming process. Proc. of the 1st Int. Symp. on FGM Forum FGM-90, Sendai, Japan. https://doi.org/10.2497/jjspm.37.922

Kaysser, W. A. (Eds.) (1999). Functionally graded materials 1998. Proceedings of the 5th international symposium on functionally gradient materials. Switzerland: Trans Tech Publications.

Koizumi, M. (1993). The concept of FGM. In Holt, J. B., Koizumi, M., Hirai, T., \& Munir, Z. A. (Eds.), Proceedings of the Second International Symposium on Functionally Gradient Materials, Ceramic Transactions, 34, 3-10. Westerville, Ohio: The American Ceramic Society.

Konda, N., \& Erdogan, F. (1994). The mixed mode crack problem in a nonhomogeneous elastic medium. Engineering Fracture Mechanics, 47(4), 533-545. https://doi.org/10.1016/0013-7944(94)90253-4

Kuang, X. et al. (2019). Grayscale digital light processing 3-D printing for highly functionally graded materials. Science Advances, 5(5), eaav5790. https://doi.org/10.1126/sciadv.aav5790

Markworth, A. J., Ramesh, K. S., \& Jr, W. P. P. (1995). Review modelling studies applied to functionally graded materials. Journal of Materials Science, 30, 2183-2193. https://doi.org/10.1007/BF01184560

Muskhelishvili, N. I. (1953). Singular Integral Equations. Groningen, The Netherlands: Noordhoff International Publishing.

Muskhelishvili, N. I. (1963). Some Basic Problems of the Mathematical Theory of Elasticity. Groningen, The Netherlands: Noordhoff International Publishing.

Sadd, M. H. (2005). Elasticity: theory, applications, and numerics. Elsevier Butterworth Heinemann.

Sneddon, I. N. (1966). Mixed Boundary Value Problems in Potential Theory. Amsterdam / New York: North-Holland Pub. Co. / Wiley.

Sneddon, I. N., \& Lowengrub, M. (1969). Crack Problems in the Classical Theory of Elasticity. New York, NY: Series title: The SIAM series in applied mathematics. Wiley.

Suresh, S., \& Mortensen, A. (1998). Fundamentals of Functionally Gradient Materials. London: The Institute of Materials.

\section{Appendix A Kernel Expressions}

$$
\begin{gathered}
K_{11}(y, \alpha)=e^{\gamma y}\left[\frac{(3-\kappa) \alpha m_{1}+i(1+\kappa) n_{1}}{4 \alpha(\kappa-1) \omega_{0}}\left[(1+\kappa) f_{32}-i \alpha(3-\kappa) f_{22}\right] e^{n_{1} y}+\frac{(3-\kappa) \alpha m_{2}+i(1+\kappa) n_{2}}{4 \alpha(\kappa-1) \omega_{0}}\left[-(1+\kappa) f_{31}+i \alpha(3-\kappa) f_{21}\right] e^{n_{2} y}\right] \\
K_{12}(y, \alpha)=e^{\gamma y}\left[\frac{(3-\kappa) \alpha m_{1}+i(1+\kappa) n_{1}}{4 \alpha(\kappa-1) \omega_{0}}(1+\kappa)\left(f_{12}-i \alpha f_{42}\right) e^{n_{1} y}-\frac{(3-\kappa) \alpha m_{2}+i(1+\kappa) n_{2}}{4 \alpha(\kappa-1) \omega_{0}}(1+\kappa)\left(f_{11}-i \alpha f_{41}\right) e^{n_{2} y}\right] \\
K_{21}(y, \alpha)=e^{\gamma y}\left[\frac{\alpha+i n_{1} m_{1}}{4 \alpha \omega_{0}}\left[(1+\kappa) f_{32}-i \alpha(3-\kappa) f_{22}\right] e^{n_{1} y}+\frac{\alpha+i n_{2} m_{2}}{4 \alpha \omega_{0}}\left[-(1+\kappa) f_{31}+i \alpha(3-\kappa) f_{21}\right] e^{n_{2} y}\right] \\
K_{22}(y, \alpha)=e^{\gamma y}\left[\frac{\alpha+i n_{1} m_{1}}{4 \alpha \omega_{0}}(1+\kappa)\left(f_{12}-i \alpha f_{42}\right) e^{n_{1} y}-\frac{\alpha+i n_{2} m_{2}}{4 \alpha \omega_{0}}(1+\kappa)\left(f_{11}-i \alpha f_{41}\right) e^{n_{2} y}\right]
\end{gathered}
$$




$$
\omega_{0}=\left(m_{1}-m_{2}\right)\left(m_{3}-m_{4}\right)\left(n_{1} n_{2}+n_{3} n_{4}\right)+\left(m_{1}-m_{4}\right)\left(m_{2}-m_{3}\right)\left(n_{2} n_{3}+n_{1} n_{4}\right)-\left(m_{1}-m_{3}\right)\left(m_{2}-m_{4}\right)\left(n_{1} n_{3}+n_{2} n_{4}\right)
$$

\section{Copyrights}

Copyright for this article is retained by the author(s), with first publication rights granted to the journal.

This is an open-access article distributed under the terms and conditions of the Creative Commons Attribution license (http://creativecommons.org/licenses/by/4.0/). 